\title{
Commentary: The Prospect of Cell-Based Therapy for Epilepsy
}

\author{
Arnold R. Kriegstein* and Asla Pitkänen ${ }^{\dagger \neq}$ \\ *Department of Neurology, University of California, San Francisco, Eli and Edythe Broad Center of Regeneration Medicine and \\ Stem Cell Research at UCSF, San Francisco, California 94143-0525, ${ }^{\dagger}$ Epilepsy Research Laboratory, A.I. Virtanen Institute for \\ Molecular Sciences, University of Kuopio, Kuopio, 70211 Finland, ${ }^{\ddagger}$ Department of Neurology, Kuopio University Hospital,
} Kuopio, 70211 Finland

Summary: About $30 \%$ of patient with epilepsy do not respond to available antiepileptic drugs. In addition to seizure suppression, novel approaches are needed to prevent or alleviate epileptogenic process after various types of brain injuries. The use of cell transplants as factories to produce endogeneous anticonvulsants or as bricks to repair abnormal ictogenic and epileptogenic neuronal circuits has generated hope that cell-based therapies could become a novel therapeutic category in the treatment arsenal of epilepsy. Herein we summarize the current status and future perspectives of cell-based therapies in the treatment of epilepsy. Key Words: Brain, disease modification, drug-refractory epilepsy, epileptogenesis, genetically-engineered cell lines, transplantation.

\section{PROGRESS TOWARD A CELL-BASED TREATMENT FOR EPILEPSY: WHERE ARE WE TODAY?}

\section{Boosting local inhibition: the case for cell transplantation in epilepsy}

Much of the current excitement surrounding stem cell research lies in the promise of cell-based approaches to the treatment of disease. Many diseases that are often discussed as potentially amenable to this approach are conditions in which a single well-defined cell type is the disease target, and replacement cells are an attractive therapeutic possibility. Examples include type 1 diabetes in which beta islet cells are deficient; Parkinson's disease in which midbrain dopamine neurons degenerate; disorders of myelination in which oligodendrocytes are lacking; and possibly amyotrophic lateral sclerosis in which motor neuron replacement is contemplated. Indeed, the notion that injured or diseased cells can be replaced has invigorated the field of regenerative medicine. Can epilepsy be a candidate disorder for cell replacement therapy? A number of lines of transgenic mice have now been characterized in which a subtype of cortical inhibitory gamma-aminobutyric acid (GABA)ergic neuron is missing and seizures are a major feature. ${ }^{1-4}$ Transplant-

Address correspondence and reprint requests to: Arnold R. Kriegstein, M.D., Ph.D., University of California, San Francisco, Eli and Edythe Broad Center of Regeneration Medicine and Stem Cell Research at UCSF, HSW-1201, 513 Parnassus Ave, San Francisco, CA 94143-0525. E-mail: kriegsteina@stemcell.ucsf.edu. ing GABAergic neurons or their precursors into the cortex of these animals could be considered a form of cell replacement. So far, however, there are few examples of human seizure disorders that are known to be due to dysfunction or lack of a specific subtype of inhibitory interneuron. One example may be patients who have mutations in the gene for alpha-thalassemia/mental retardation syndrome X-linked (ATRX), a chromatin remodeling protein. This mutation causes a syndrome characterized by severe cognitive deficits, microcephaly, and epilepsy among other features. ${ }^{5}$ When the ATRX gene was conditionally inactivated in the forebrain of mice, a severe reduction in GABAergic interneurons was observed in the cortex, ${ }^{6}$ a finding that could account for many of the nervous systems manifestations of the human disease, including seizures. Spreafico and colleagues have also shown interneuron loss in focal cortical dysplasia associated with epilepsy. ${ }^{7}$ However, seizures themselves may cause selective loss of GABAergic neurons in the hippocampus or amygdala, a finding that has been well documented to be a consequence of status epilepticus (SE) in animal models (for example ${ }^{8,9}$ ). Examination of hippocampal tissue resected from patients with temporal lobe epilepsy has also demonstrated loss of subpopulations of GABAergic neurons, ${ }^{10}$ but whether this is the primary cause of the underlying seizure disorder or a consequence of seizures is still debated.

The causes of epilepsy are numerous, and in most cases they are not understood. Many of the genetic disorders associated with epilepsy in humans can be characterized as 
"channelopathies" that produce changes in membrane excitability or result from mutations in transmitter receptors or synaptic proteins. ${ }^{11}$ These disorders have as a common consequence, either an increase in neuronal excitability or attenuation of inhibition. Given that some anti-epileptic drugs enhance GABA-mediated inhibition, boosting local inhibition in the brain or cortex could be an effective mode of treatment in carefully selected cases. In cases of intractable focal epilepsy, the use of a cell-based approach to inhibit local circuit hyperactivity is a potentially attractive strategy. However, even in disorders in which excitability is globally disordered, focal treatment may be helpful. For example, the pars reticulata, a subregion of the substantia nigra, is thought to participate as a gate for the generalization of seizure activity. ${ }^{12}$ Lesioning this region, or enhancing local GABAergic function, can produce anticonvulsant effects. Therefore, in addition to focal cortical epilepsy, it may be possible to treat generalized seizures using localized, cell-based therapy.

\section{Non-GABAergic fetal cells}

A key advantage of a cell transplant approach over drug therapy is that cell treatment can be locally restricted, whereas drugs can have widespread and systemic adverse effects. The first attempts at cell therapy for epilepsy used fetal noradrenergic neurons derived from the rat locus coeruleus that were grafted bilaterally to the hippocampus of adult rats following chemical lesioning of the central catecholamine pathway. ${ }^{13,14}$ The noradrenergic neurons were presumed to exert tonic inhibitory effects ${ }^{15}$ and were able to suppress the development of kindling-induced seizures. However, adrenergic grafts did not suppress kindling in nonlesioned animals and did not suppress seizures if grafted after kindling. Fetal catecholamine-releasing neuron transplants have been shown to be moderately effective at suppressing a variety of seizures in animal models. Intraventricular grafting of fetal locus coeruleus or hippocampal neurons has mild beneficial effects on seizure frequency after kainic acid (KA) induced chronic epilepsy, ${ }^{16}$ and it also has modest effects on seizure duration in the audiogenic seizure prone rat. ${ }^{17}$ Grafting fetal serotonergic or cholinergic neurons has also been shown to suppress seizures in epilepsy-prone animals that have had either their serotoninergic or cholinergic pathways, respectively, lesioned. ${ }^{18-20}$ Whether serotoninergic or cholinergic neuron grafts would be effective in other epilepsy models is uncertain, and lesions of catecholamine or cholinergic pathways are not commonly found in human epilepsy. ${ }^{21}$ Transplanting fetal hippocampal neurons, but not neocortical neurons, bilaterally to the hippocampus in kindled rats has been reported to shorten seizure episodes, but the mechanism is unknown and the effects were not longlasting. ${ }^{22}$

\section{Genetically-engineered cell lines}

Another approach, based on the observation that adenosine has anticonvulsant and neuroprotective properties, has been to use adenosine-releasing cells as a means to focally deliver adenosine to an epileptogenic brain region. Synaptic adenosine is regulated by the adenosine metabolizing enzyme, adenosine kinase (ADK), which is present in glial cells. The "ADK hypothesis of epileptogenesis" proposes that brain injury leads to astrogliosis and upregulation of ADK activity, thus producing a focal adenosine deficiency and epileptogenesis. ${ }^{23}$ Boison and colleagues have pursued a series of cell-based therapeutic strategies to prevent epileptogenesis based on the ADK hypothesis. The first attempt was based on rodent fibroblasts engineered to release adenosine that were encapsulated in a semi-permeable polymer membrane to prevent immune rejection. Intraventricular grafting transiently reduced kindled seizures in rats, but the encapsulated cells did not survive long. ${ }^{24}$ In subsequent experiments, mouse embryonic stem cell-derived neural precursor cells were used, after they had been engineered to release adenosine. These cells survived well and attenuated kindling in grafted animals, ${ }^{25}$ and also protected against the development of spontaneous seizures when grafted 24 hours after intrahippocampal KA injection. ${ }^{26}$ Although this approach is still a long way from possible clinical application, it is likely that use of a genetically engineered cell line will present an even higher barrier to regulative approval than would a genetically unaltered cell line.

\section{Mesenchymal stem cells}

Human mesenchymal stem cells obtained from the bone marrow have attracted interest as potential autologous cell therapy agents because they could be grafted without triggering an immune response. As reported by Detlev Boison in this issue of Neurotherapeutics, human mesenchymal stem cells (hMSCs) have been engineered to release adenosine through micro-RNA directed knockdown of the adenosine-metabolizing enzyme, ADK. The micro-RNA was introduced using a lentiviral vector, and the hMSCs release adenosine after transplantation. ADK-knockdown hMSCs were able to reduce acute KAinduced seizures and to improve neuron survival when grafted to the hippocampus one week prior to KA-induced seizures. These results suggest that adenosinereleasing hMSCs may have anti-epileptogenic potential. However, when the cells were grafted one day after KA injection, even though the frequency and duration of spontaneous seizures occurring three weeks later were reduced, this effect was reversed with an adenosine receptor antagonist, suggesting that the underlying process leading to the development of epilepsy (epileptogenesis) was not altered by the presence of the grafted cells. Thus, for anti-epileptogenic effects, the timing of the graft appears key, and even though chronic seizures develop over the weeks after KA injection, unless adenosinereleasing hMSCs are present at the time of acute KA- 
induced SE, the underlying seizure producing events appear not to be modified by hMSC grafts. It would obviously not be possible to treat patients in advance of an epileptogenic insult, but adenosine-releasing hMSCs may nonetheless be able to attenuate pre-existing focal seizure activity.

\section{Fetal GABAergic cells}

Possibly the first study to graft fetal GABAergic neurons in an effort to treat pilocarpine-induced seizures used cells derived from the ganglionic eminence of E16 rats. ${ }^{27}$ However, benefit was also observed with control cells from the sciatic nerve, suggesting that the effect may have resulted from lesioning of the substantia nigra during grafting. ${ }^{27}$ Grafting fetal GABAergic cells to substantia nigra produced transient anticonvulsant effects in kindled rats, ${ }^{28}$ as did grafting of an immortalized cortical cell line engineered to produce GABA. ${ }^{29}$ Variations on this approach have included pretreatment of fetal cells with fibroblast growth factor- 2 and a caspase inhibitor in attempts to increase the number of surviving cells and extend their lifetime in the host brain. ${ }^{30}$ In these experiments cells were injected bilaterally into the hippocampi four days after KA-induced SE and the frequency of spontaneous seizure-like behaviors was significantly reduced compared to sham controls as long as one year later.

In 1998, Diacrin Inc. initiated a trial using fetal porcine GABAergic cell grafts in three patients with medically refractory epilepsy. ${ }^{31}$ Seizure frequency reportedly decreased, but the Food and Drug Administration halted the trial over safety concerns. ${ }^{32}$ Experiments have recently demonstrated that inhibitory interneurons derived from the embryonic medial ganglionic eminence can migrate from the injection site and integrate into the cortex of adult rats. ${ }^{33}$ These cells also increase local inhibition in the adult brain and may be able to limit the initiation or spread of seizure discharges.

\section{Genetically-engineered GABAergic cells}

An alternative to the use of fetal GABAergic neurons, is the use of genetically-engineered GABA-producing cells, as described by Thompson in this issue of Neurotherapeutics. Several cell lines have been engineered to enhance their potential to serve as GABA "pumps" when grafted into focal epileptic brain regions. Either GABAergic or non-GABAergic neural progenitor cells have been immortalized and made to overexpress the GABA synthetic enzymes, GAD67 or GAD65. ${ }^{29,34}$ In addition, cell lines were engineered with a temperaturesensitive oncogene so that cell division could be regulated, and also engineered to express the GAD65 transgene under the control of a tetracycline-off promoter so that GABA release could also be controlled. When these cells were transplanted into the substantia nigra of chronically seizing (KA-treated) animals, the frequency of spontaneous seizures was reduced, but not if animals also received doxycycline to turn off GAD65 expression. ${ }^{35}$ However, the long-term survival of the engineered cell lines remains a limiting factor. The possibility of generating GABA-producing cells, possibly also engineered for regulated GABA release from stem cells may provide a solution to the problems of integration and survival. Indeed, Thompson describes genetically engineered GABA-producing cells that are generated from stem cells, and reports that when grafted bilaterally to the hippocampi the cells appear to suppress spontaneous seizures. Although this is encouraging, the use of genetically-engineered cell lines will present an additional hurdle to regulative approval.

\section{HOW FAR ARE WE FROM APPLYING EXPERIMENTAL CELL THERAPY TO THE TREATMENT OF HUMAN EPILEPSY?}

\section{Candidate indication areas}

Approximately $30 \%$ of patients with epilepsy are drug refractory and do not respond to current treatments. ${ }^{36}$ Novel approaches are needed, and as previously noted, many of the cell-based therapies applied in experimental models suppress evoked and/or spontaneous seizures in a variety of seizure models, including rats with audiogenic seizures, evoked seizures in kindling models, or spontaneous seizures that develop within days to weeks after SE. The anticonvulsant effect of cell therapies is believed to relate to either the increased availability of anticonvulsant substances like GABA or adenosine in the focal area, ${ }^{23,35}$ or the "normalization" of abnormal ictogenic circuitry in the hippocampus. ${ }^{30,37}$ The initial experiments are promising, but larger preclinical studies are needed to demonstrate the percentage of animals that respond to treatment; "who" the responders are (e.g., lesion type and severity); the long-term efficacy; possible adverse events; and co-morbid effects (e.g., altered memory processing). In particular, if the cell therapies are presumed to provide a "cure" by normalizing ictogenic circuitry, more experiments are necessary to expand the studies from SE models to other acquired and genetic etiologies with different cellular alterations to examine how broadly this approach might work and, in particular, how likely it might apply in cases where the etiology is unknown.

Another indication is the prevention of epileptogenesis, for example, after traumatic brain injury. A large number of cell transplantation studies have demonstrated enhanced motor and cognitive recovery after experimental traumatic brain injury. ${ }^{38}$ The proposed mechanisms are multiple, varying from repair of circuitry to increase in availability of neurotrophic and other factors to facilitate self-repair process in the damaged brain area. Unfortunately, none of these experiments investigated "epilepsy" as an outcome measure. Studies have investigated 
the efficacy of cell therapies on epileptogenesis in the kindling model and in models in which spontaneous seizures develop after SE. Interpretation of the data from these experiments has been difficult. The suppression of a kindling stimulus by the transplant can affect the kindling process. In SE models, it becomes critical to demonstrate the lack of effect of transplantation on the "initial epileptogenic insult," that is, SE itself. Alleviation of SE results in prolongation of latency to the appearance of the first spontaneous seizure, and to lower seizure frequency (disease modification). Even if transplantation is post-injury, the question remains of how to differentiate the anticonvulsant effect from a true antiepileptogenic effect. This question is difficult to resolve unless the cell graft is removed or inactivated. Also, it is unknown for how long the animals should be followed by video-EEG monitoring to demonstrate anti-epileptogenesis (no seizures), or even disease modification. Finally, use of SE models present a challenge for any candidate anti-epileptogenic treatment because of the possibility of false negative findings related to the robust damage produced in this model. Therefore, studies in SE models in which the severity of SE is reduced, or use of models with more limited brain damage (e.g., stoke, traumatic brain injury models) might be more sensitive. Finally, guidelines are needed to define what preclinical evidence will be required to demonstrate an anti-epileptic or anti-epileptogenic effect of cell therapy prior to initiating a clinical trial.

\section{Availability, cost, and ethical issues related to the use of cell therapy in the treatment of epileptogenesis and epilepsy}

Like any new technology, cell-based therapy is associated with many practical problems that will need to be overcome before cell treatment would be part of the treatment arsenal for epilepsy. These include availability of embryonic or other starting tissue, safety issues related to engineering of viral constructs and their transduction, production of cells under GMP conditions, targeted differentiation of transplanted cells, graft rejection, tumor formation, and legislative and ethical issues. Thorny as some of these issues may be, progress is already being made in all of these problem areas. Solutions could include ex vivo manipulation of grafts toward an appropriate stage of lineage commitment, use of autologous cell transplants, use of transplanted cells as delivery vehicles for drug or gene therapy, patient-specific induced cells, and engineering of transplants that can be removed by suicide gene inserts. Cell-based therapies are unlikely to become the mainstays of treatment for most epilepsies any time soon, but in cases of medically-intractable seizures, and especially in cases in which surgery may not be an option, such an approach (if perfected) could well address a currently unmet need.

\section{REFERENCES}

1. Cobos I, Calcagnotto ME, Vilaythong AJ, et al. Mice lacking Dlx1 show subtype-specific loss of interneurons, reduced inhibition and epilepsy. Nat Neurosci 2005;8:1059-1068.

2. Sarkisian MR, Frenkel M, Li W, Oborski JA, LoTurco JJ. Altered interneuron development in the cerebral cortex of the flathead mutant. Cereb Cortex 2001;11:734-743.

3. Powell EM, Campbell DB, Stanwood GD, et al. Genetic disruption of cortical interneuron development causes region- and GABA cell type-specific deficits, epilepsy, and behavioral dysfunction. J Neurosci 2003;23:622-631.

4. Butt SJ, Sousa VH, Fuccillo MV, et al. The requirement of Nkx2-1 in the temporal specification of cortical interneuron subtypes. Neuron 2008;59:722-732.

5. Gibbons RJ, Higgs DR. Molecular-clinical spectrum of the ATR-X syndrome. Am J Med Genet 2000;97:204-212.

6. Seah C, Levy MA, Jiang Y, et al. Neuronal death resulting from targeted disruption of the Snf2 protein ATRX is mediated by $\mathrm{p} 53$. J Neurosci 2008;28:12570-12580.

7. Garbelli R, Meroni A, Magnaghi G, et al. Architectural (Type IA) focal cortical dysplasia and parvalbumin immunostaining in temporal lobe epilepsy. Epilepsia 2006;47:1074-1078.

8. Knopp A, Frahm C, Fidzinski P, Witte OW, Behr J. Loss of GABAergic neurons in the subiculum and its functional implications in temporal lobe epilepsy. Brain 2008;131:1516-1527.

9. Tuunanen J, Halonen T, Pitkanen A. Status epilepticus causes selective regional damage and loss of GABAergic neurons in the rat amygdaloid complex. Eur J Neurosci 1996;8:2711-2725.

10. de Lanerolle NC, Kim JH, Robbins RJ, Spencer DD. Hippocampal interneuron loss and plasticity in human temporal lobe epilepsy. Brain Res 1989;495:387-395.

11. Noebels JL. The biology of epilepsy genes. Annu Rev Neurosci 2003;26:599-625.

12. Faingold CL. Emergent properties of CNS neuronal networks as targets for pharmacology: application to anticonvulsant drug action. Prog Neurobiol 2004;72:55-85.

13. Barry DI, Kikvadze I, Brundin P, Bolwig TG, Bjorklund A, Lindvall $\mathrm{O}$. Grafted noradrenergic neurons suppress seizure development in kindling-induced epilepsy. Proc Natl Acad Sci U S A 1987:84:8712-8715.

14. Barry DI, Wanscher B, Kragh J, et al. Grafts of fetal locus coeruleus neurons in rat amygdala-piriform cortex suppress seizure development in hippocampal kindling. Exp Neurol 1989; 106:125-132.

15. Bjorklund A, Lindvall O. Cell replacement therapies for central nervous system disorders. Nat Neurosci 2000;3:537-544.

16. Holmes GL, Thompson JL, Huh K, Holmes C, Carl GF. Effect of neural transplants on seizure frequency and kindling in immature rats following kainic acid. Brain Res Dev Brain Res 1991;64:47-56.

17. Holmes GL, Thompson JL, Huh K, Stuart JD, Carl FG. Effects of neural transplantation on seizures in the immature genetically epilepsy-prone rat. Exp Neurol 1992;116:52-63.

18. Clough RW, Browning RA, Maring ML, Statnick MA, Wang C, Jobe PC. Effects of intraventricular locus coeruleus transplants on seizure severity in genetically epilepsy-prone rats following depletion of brain norepinephrine. J Neural Transplant Plast 1994;5:65-79.

19. Clough R, Statnick M, Maring-Smith M, et al. Fetal raphe transplants reduce seizure severity in serotonin-depleted GEPRs. Neuroreport 1996;8:341-346.

20. Ferencz I, Kokaia M, Elmer E, Keep M, Kokaia Z, Lindvall O. Suppression of kindling epileptogenesis in rats by intrahippocampal cholinergic grafts. Eur J Neurosci 1998;10:213-220.

21. Bjorklund A, Segal M, Stenevi U. Functional reinnervation of rat hippocampus by locus coeruleus implants. Brain Res 1979;170: 409-426.

22. Miyamoto O, Itano T, Yamamoto Y, et al. Effect of embryonic hippocampal transplantation in amygdaloid kindled rat. Brain Res 1993;603:143-147.

23. Boison D. The adenosine kinase hypothesis of epileptogenesis. Prog Neurobiol 2008;84:249-262. 
24. Huber A, Padrun V, Deglon N, Aebischer P, Mohler H, Boison D. Grafts of adenosine-releasing cells suppress seizures in kindling epilepsy. Proc Natl Acad Sci U S A 2001;98:7611-7616.

25. Li T, Steinbeck JA, Lusardi T, et al. Suppression of kindling epileptogenesis by adenosine releasing stem cell-derived brain implants. Brain 2007;130:1276-1288.

26. Li T, Ren G, Lusardi T, et al. Adenosine kinase is a target for the prediction and prevention of epileptogenesis in mice. J Clin Invest 2008; 118:571-582.

27. Fine A, Meldrum BS, Patel S. Modulation of experimentally induced epilepsy by intracerebral grafts of fetal GABAergic neurons. Neuropsychologia 1990;28:627-634.

28. Loscher W, Ebert U, Lehmann H, Rosenthal C, Nikkhah G. Seizure suppression in kindling epilepsy by grafts of fetal GABAergic neurons in rat substantia nigra. J Neurosci Res 1998;51:196-209.

29. Thompson K, Anantharam V, Behrstock S, Bongarzone E, Campagnoni A, Tobin AJ. Conditionally immortalized cell lines, engineered to produce and release GABA, modulate the development of behavioral seizures. Exp Neurol 2000;161:481-489.

30. Hattiangady B, Rao MS, Shetty AK. Grafting of striatal precursor cells into hippocampus shortly after status epilepticus restrains chronic temporal lobe epilepsy. Exp Neurol 2008;212:468-481.

31. Edge AS. Current applications of cellular xenografts. Transplant Proc 2000;32:1169-1171.
32. Loscher W, Gernert M, Heinemann U. Cell and gene therapies in epilepsy - promising avenues or blind alleys? Trends Neurosci 2008;31:62-73

33. Alvarez-Dolado M, Calcagnotto ME, Karkar KM, et al. Cortical inhibition modified by embryonic neural precursors grafted into the postnatal brain. J Neurosci 2006;26:7380-7389.

34. Castillo CG, Mendoza S, Freed WJ, Giordano M. Intranigral transplants of immortalized GABAergic cells decrease the expression of kainic acid-induced seizures in the rat. Behav Brain Res 2006;171: 109-115.

35. Thompson KW, Suchomelova LM. Transplants of cells engineered to produce GABA suppress spontaneous seizures. Epilepsia 2004; 45:4-12.

36. Kwan P, Brodie MJ. Early identification of refractory epilepsy. N Engl J Med 2000;342:314-319.

37. Shetty AK, Zaman V, Hattiangady B. Repair of the injured adult hippocampus through graft-mediated modulation of the plasticity of the dentate gyrus in a rat model of temporal lobe epilepsy. J Neurosci 2005;25:8391-8401.

38. Pitkänen A, Longhi L, Marklund N, Morales D, McIntosh TK. Mechanisms of neuronal death and neuroprotective strategies after traumatic brain injury. Drug Discov Today Dis Mech 2005;2:409-418. 